\title{
Motor resonance is sensitive to long but not short modulations of physical exercise
}

\begin{abstract}
The aim of this study is to evaluate the mutual effect experience in a type of sport and the exertion of various levels of physical exercise have on the ability to recognize and decode others' facial expressions. Twenty-one taekwondo athletes and 17 soccer players participated in this study. Each of these participants performed a facialrecognition task while running on treadmill, with the task being performed at five different running speeds (i.e., at rest, $60 \%, 80 \%, 100 \%$, and $120 \%$ of maximal aerobic speed). The results of this study revealed that the participants' motor resonance increased with greater effort produced. It also revealed that the practitioners of the fighting sport (taekwondo) were systemically faster and more accurate in terms of recognizing facial expressions than the practitioners of the team sport (soccer). The fighters' advantage over the collective sports practitioners in regard to recognition performance was mainly present at conditions involving higher intensities of effort. In conclusion, our study reveals that expertise in fighting sports, which require precise decoding of opponents' emotional states, is associated with enhanced ability to recognize facial expressions.
\end{abstract}

Keywords: facial recognition, neural plasticity, physical effort, soccer, taekwondo
Volume I Issue 5 - 2017

\author{
Adel Jouini, ${ }^{1,2}$ Bessem Mkaouer, ${ }^{3}$ Karim \\ Chamari $^{4}$ \\ 'High Institute of Education, Virtual University of Tunis, Tunisia \\ 2Sports Performance Optimization, National Center of Medicine \\ and Science in Sports (CNMSS), Tunisia \\ ${ }^{3}$ Higher Institute of Sport and Physical Education of Ksar Saïd, \\ Manouba University, Tunisia \\ ${ }^{4} \mathrm{AHP}$ Research Center, Doha, Qatar
}

Correspondence: Bessem Mkaouer, Higher Institute of Sport and Physical Education of Ksar Saï, Manouba University, Tunisia, Tel+216-230667l6,Email bessem_gym@yahoo.fr

Received: October 07, 2017 | Published: November 10, 2017
Abbreviations: MNS, mirror neuron system; TKD, taekwondo athletes; SOC, soccer players; CRT, choice reaction time; MAS, maximal aerobic speed; E, effort; E60, effort at $60 \%$ of MAS; E80, effort At $80 \%$ of MAS; E100, effort at $100 \%$ of MAS; E120, effort at $120 \%$ of MAS; RT, reaction time; OEE, observed exerted effort; SD, standard deviation; FMRI, functional magnetic resonance imaging

\section{Introduction}

There are reported beneficial and lasting effects of physical exercise on cognitive function with increased blood flow to the brain and neurotransmitter levels, enhanced plasticity and develop brain volume. ${ }^{1}$ Moreover, it has been shown that physical activity can lead to a better concentration, attention and information processing. ${ }^{2}$ Recent neurophysiological studies shown that the mirror neuron system (MNS) allows an individual to determine or simulate not only another individual's intentions, but also their state of mind, which facilitates the performance of appropriate interactions with the observed agent. ${ }^{3-5}$ During processing of the emotions shown through an actor's facial expressions, mirror neurons fire and provoke an internal simulation of the observed motor behavior, which in turn evokes a similar emotion in the observer's mind; this phenomenon is known as motor resonance. ${ }^{6,7}$ Motor resonance can be influenced by the short-term effects of physical exercise, such as those related to acute intensification of effort. It has been well documented that when an observer's level of effort is increased, many cognitive functions are enhanced; ${ }^{8-11}$ this enhancement is commonly attributed to an increase in the level of arousal as a result of the intensification of the physical effort. ${ }^{12}$ Further, this effort exerted by the observer may in turn induce facial movements that are congruent with the facial expressions exhibited by the actor. Theories concerning embodied cognition ${ }^{13,5}$ suggest that enhancement of recognition processes occurs when the facial expressions of an observer match those exhibited by an actor During sporting competitions, the intensity of the effort athletes produce varies with the strategy employed and their physical capacity. Since the status of the athlete himself (their own fatigue) can influence their ability to recognize the facial expressions of their opponent (the actor), ${ }^{10,11}$ it is plausible that this ability will also be affected by the intensities of effort produced during the competition. In short, in this paper we wish to examine whether modifying the level of intensity of physical exercise can have an impact on observers' ability to decode facial expressions associated with producing physical effort.

Motor resonance has also been shown to be sensitive to long-term influences, such as expertise in a particular activity; ${ }^{14,15}$ further, motor resonance is crucial in some sporting activities, particularly those that require direct evaluation of an opponent's status. For instance, higher performance in fighting sports (i.e., taekwondo) relies on the ability of each athlete to accurately estimate the level of effort or fatigue his/her opponent is experiencing. This information can be used to efficiently manage the effort produced and to choose an appropriate fighting strategy in response. Supporting this assumption, a study by CalvoMerino et al. ${ }^{14}$ mentioned that motor resonance is highly influenced by specific expertise in a particular physical activity. Another important finding to note is that motor resonance is increased by a higher amount when experts in a sport observe a short sequence of movement that is common in their domain of expertise than when they observe a sequence of unusual activity. ${ }^{16,17}$ Moreover, Meir et al. ${ }^{16}$ revealed that extensive practice in a specific sport can influence brain plasticity through the modification of the activities of the motor areas solicited by that sport. ${ }^{18}$ Considering the above, the present study evaluates whether expertise in fighting-sports (e.g., taekwondo), in which facial recognition represents a strategic ability, produces better recognition of facial expressions than expertise in sports in which such ability is less important (e.g., soccer). ${ }^{19,20}$ The present study aims to evaluate both the short-term (effect of level of effort produced) and longterm (expertise in a specific sport) effects of physical activities on the ability to recognize facial expressions associated with producing 
different levels of physical effort. An additional investigation here was to verify whether the impact of the short-term effect is modulated by the impact of the long-term effect.

\section{Methods}

\section{Participants}

Twenty-one expert taekwondo athletes (TKD, age $22 \pm 1$ years; height $1.70 \pm 0.07 \mathrm{~m}$; body mass $70.1 \pm 7.5 \mathrm{~kg}$ ) and 17 soccer players (SOC, age $23 \pm$ lyears; height $1.70 \pm 0.03 \mathrm{~m}$; body mass $72.9 \pm 3.7 \mathrm{~kg}$ ) participated in this study. Only TKD and SOC players with at least 10 years of experience in their respective sports and who participate regularly in national and international competition were included. Further, all participants were right-handed and possessed normal vision. All participants were volunteers and were informed of the procedures, methods, benefits, and possible risks involved in the study, and a written consent form was obtained from each. The experimental protocol was performed in accordance with the Declaration of Helsinki concerning human experimentation and was approved by the local ethical committee (decision number: 052/17). The experiment was conducted during the regular sports season, four to five months after the start of the competitive period; the participants were asked to maintain their regular training schedules throughout the experimental period (Table 1).

Table I Participants biological parameters

\begin{tabular}{|c|c|c|c|c|c|c|c|c|c|c|c|c|c|c|}
\hline Sport & $\mathbf{N}$ & YP & Age & Height & $\begin{array}{l}\text { Body } \\
\text { mass }\end{array}$ & VO2 max & MAS & $\begin{array}{l}\text { HR } \\
\text { rest }\end{array}$ & $\begin{array}{l}\text { HR } \\
\max \end{array}$ & $\begin{array}{l}\text { HR } \\
\text { e60 }\end{array}$ & $\begin{array}{l}\text { HR } \\
\text { e80 }\end{array}$ & $\begin{array}{l}\text { HR } \\
\text { el } 100\end{array}$ & $\begin{array}{l}\text { HR } \\
\text { el } 20\end{array}$ & $\begin{array}{l}\text { La } \\
\text { vo2max }\end{array}$ \\
\hline TKD & 21 & $\begin{array}{l}11.3 \pm \\
1.2\end{array}$ & $\begin{array}{l}22.1 \pm \\
1.5\end{array}$ & $\begin{array}{l}178.4 \pm \\
8.5\end{array}$ & $\begin{array}{l}70.25 \pm \\
9.2\end{array}$ & $56.87 \pm 5.5$ & $\begin{array}{l}16.25 \pm \\
1.5\end{array}$ & $53 \pm 2$ & $193 \pm 4$ & $\begin{array}{l}115 \pm \\
5\end{array}$ & $150 \pm 6.5$ & $190 \pm 4.5$ & $191 \pm 5$ & $12.7 \pm 1.1$ \\
\hline SC & 17 & $\begin{array}{l}10.9 \pm \\
0.7\end{array}$ & $\begin{array}{l}23.2 \pm \\
1\end{array}$ & $\begin{array}{l}177.8 \pm \\
6.5\end{array}$ & $\begin{array}{l}72.99 \pm \\
7\end{array}$ & $56.62 \pm 5.25$ & $\begin{array}{l}16.18 \pm \\
1.5\end{array}$ & $53 \pm 3$ & $192 \pm 4$ & $\begin{array}{l}120 \pm \\
16\end{array}$ & $155 \pm 10$ & $188 \pm 6.5$ & $189 \pm 5.5$ & $13.77 \pm 2.05$ \\
\hline
\end{tabular}

$\mathrm{N}$, number of participants; YP, years of practice; TKD, Taekwondoist; SC, Soccer; MAS, maximum aerobic speed; HR, heart rate; E60, effort equivalent to $60 \%$ of MAS; E80, effort equivalent to $80 \%$ of MAS; EI00, effort equivalent to $100 \%$ of MAS; EI20, effort equivalent to $120 \%$ of MAS; La VO ${ }_{2 \text { max }}$, blood acid lactic concentration A

\section{Experimental design and procedures}

\section{Physiological measures}

The participants underwent seven assessment sessions. In the first session, participants were provided complete information concerning the equipment and testing procedures that would be used and gave their consent to participate to this study; we also recorded their anthropomorphic and demographic data at this point. In session 2 , participants were required to perform a VO2 max test, which was conducted by following the protocol described by Harling et al. ${ }^{18}$ This was performed in order to determine each participant's maximal aerobic speed (MAS) in this study, MAS is defined as the highest speed reached and sustained for one minute during the VO2 max test.

In sessions 3-7 the participants performed facial-recognition choice reaction time (CRT) tasks while running on a treadmill. The tasks were performed while the participants were exerting five different levels of effort (E); rest (participant did not move), E60 (60\% of MAS), E80 ( $80 \%$ of MAS), E100 (100\% of MAS), and E120 (120\% of MAS). In each stage, MAS was controlled by monitoring speed and heart rate, and the order of the five conditions was counterbalanced (Latin square).

The categorization of these levels of effort was based on the specificity of the efforts, naturally it would be difficult for the participants to maintain a maximum (E100) or a supramaximal (E120) intensity of their MAS for more than two minutes. ${ }^{21,22}$

\section{Behavioral measures}

The task also involved the participants being shown 40 pictures of facial expressions, with 20 depicting moderate physical effort and 20 depicting intensive physical effort. The participants had one minute to reach the target speed for the level, at which point the task began automatically, lasting two minutes for each effort level, as mentioned above. The participants' task was to indicate whether the faces displayed on the screen were expressing moderate or intensive physical effort. The order of the stimuli was pseudorandom. The instructions given to the TKD and SOC groups emphasized both speed and accuracy, and the participants did not receive feedback about the accuracy of their responses. Each image was shown for three seconds (Figure 1); each was preceded by a fixation cross $(+)$ displayed in the center of the screen (for 750 to $1000 \mathrm{~ms}$ ), and this was immediately followed by a facial image stimulus. The image remained visible until the participants responded or if the response time exceeded $1000 \mathrm{~ms}$; responses that were provided after this fixed duration were omitted from the analysis. The trial ended with a white screen, which served as an inter-trial interval (lasting from 1000 to $1250 \mathrm{~ms}$ ).

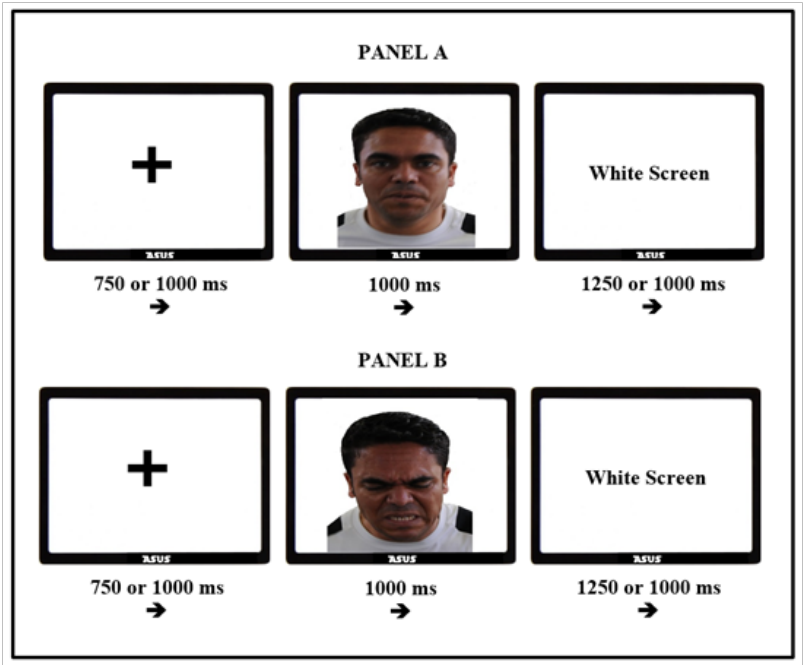

Figure I A schematic representation of the sequence of events in a trial involving a picture of a low-intensity-effort facial expression (panel A) and that of a high-intensity-effort facial expression (panel B). Arrows indicate the direction of the evolution of the events. 
The stimuli used were color images (with dimensions of $17 \mathrm{~cm} \times 12 \mathrm{~cm}$ ) depicting various males' faces expressing different facial expressions associated with different intensities of physical effort. These images were obtained through the performance of a process in which samples of 10 participants (mean age $25.1 \pm 1.2$ years) were required to perform moderate- (60\% and $80 \%$ of MAS) or highintensity exercise $(100 \%$ and $120 \%$ of MAS). Photos were taken when participants had produced the required level of effort for 90 seconds. A total of 40 photos (i.e., 10 participants expressing four levels of effort) were then used as stimuli for the trials discussed in this paper.

Stimuli presentation and response collection was conducted using the 2014 software Inquisit4® version 4.0.5.0 (Millisecond Software ${ }^{\circledR}$ LLC) ${ }^{23}$ and the stimuli were presented on a 17 -inch, highresolution laptop screen (an Asus ${ }^{\circledR} \mathrm{N} 76 \mathrm{~V}$ ); this screen was fixed on an adjustable support placed in front of the participant's head and at a viewing distance of $60 \mathrm{~cm}$. Responses to stimuli were made using the dominant hand by pressing one of the two buttons on a $3 \mathrm{M}^{\circledR}$ ergonomic mouse (ref. EM550GPS) that was fixed to the ramp of the treadmill. The assignment of critical stimuli to response buttons was counterbalanced across participants (Figure 1).

\section{Statistical analyses}

Data are reported as mean \pm standard deviations (SD). All variables were tested for normality using the Shapiro-Wilk test and the results of this showed that the data were normally distributed. The obtained data (accuracy and reaction time (RT)) then underwent a three wayANOVA with Sport Expertise (fighting vs. collective) as a grouping

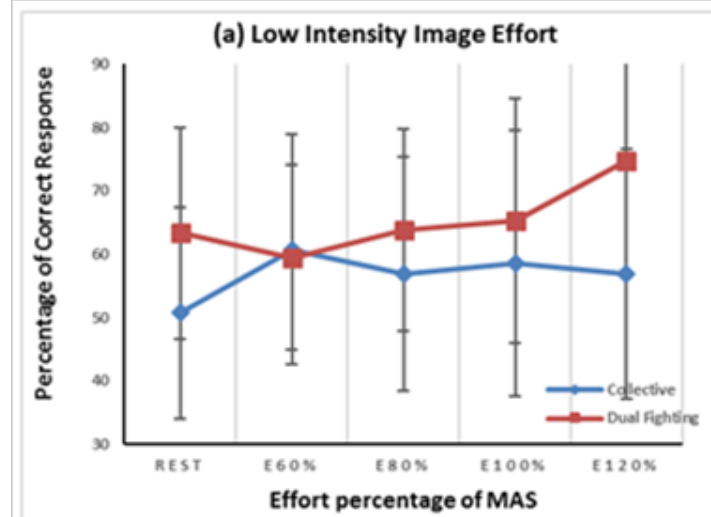

factor, and Effort-related Facial Expression (moderate vs. high intensity) and Observed Exerted Effort (OEE) (rest, E60\%, E80\%, $\mathrm{E} 100 \%$ and $120 \%)$ as repeated measure factors. Significance was set at $0.05 \%(\mathrm{p} \leq 0.05)$. Calculations and statistical analyses were performed using SPSS 20.0 (SPSS, Inc, Chicago, IL, USA).

\section{Results}

\section{Physiological measures}

The results of the ANOVA of the biological measures showed no significant difference in VO2 max, MAS, HR max and LaVO2 max between the two groups (Table 1).

\section{Response accuracy}

The percent of correct responses as a function of the manipulated factors is depicted in Figure 2. The analysis revealed that the fighting sport group $(\mathrm{F}(1,36)=30.88, \mathrm{p}<0.001, \omega=0.99)$ interpreted facial expressions significantly more accurately $(\mathrm{M}=69 \% ; \mathrm{SD}=17 \%)$ than the collective sport group $(59 \%, \mathrm{SD}=17 \%)$. Additionally, overall, among the fighting group, facial expressions relating to higher intensity effort $(\mathrm{M}=68 \%, \mathrm{SD}=18 \%)(\mathrm{F}(1,36)=13.78, p<0.001, \hat{\omega}=0.95)$ were significantly better recognized than moderate-physical-effort facial expressions ( $\mathrm{M}=67 \%, \mathrm{SD}=18.5 \%)$. The level of accuracy also varied significantly as a function of $\operatorname{OEE}(\mathrm{F}(4,144)=8.50, \mathrm{p}<0.001, \dot{\omega}=0.95)$. As can be seen in Figure 2, the highest level of accuracy was obtained with the $\mathrm{E} 120 \%$ condition $(\mathrm{M}=70 \%, \mathrm{SD}=17 \%$ ) and the lowest was found with the rest condition ( $\mathrm{M}=54 \%, \mathrm{SD}=18 \%)$. Intermediary values were obtained for $\mathrm{E} 60 \%$, E $80 \%$ and $\mathrm{E} 100 \%$ (Figure 2).

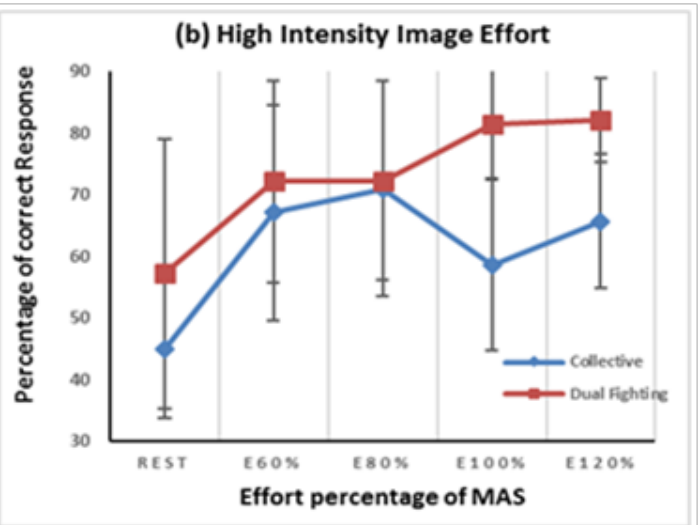

Figure 2 Variation of the mean percent of correct response as a function of the type of sport expertise (fighting versus collective sport) and as a function of the intensity of the physical exercise $(E)$ intensity (from rest to $120 \%$ MAS).

The factors of Effort Expression and Sport Expertise did not interact significantly $(\mathrm{F}(1,36)<1, \mathrm{NS})$; however, the analysis revealed a significant dual interaction between Sport Expertise and OEE (F(4, 144) $=2.7, p<0.05, \omega=0.73)$ and between Effort Expression and $\operatorname{OEE}(\mathrm{F}(4,144)=4.1, \mathrm{p}<0.001, \omega=0.92)$. Finally, the analysis did not reveal a triple interaction $(\mathrm{F}(4,144)=1.5, \mathrm{p}>0.1)$. As can be seen in (Figure 2), the dual interaction between Effort Expression and OEE indicates that the rate of correct recognition increased with increases in the intensity of the effort exerted by the participants; however, the magnitude of the increase was more significant for the highintensity facial images (rest $=51 \%, \mathrm{SD}=18 ; \mathrm{E} 120 \%=78 \%, \mathrm{SD}=12 \%$, $\mathrm{d}=17 \%$ ) than for the moderate-intensity facial images (Rest $=58 \%$, $\mathrm{S}=17 ; \mathrm{E} 120 \%=66 \%, \mathrm{SD}=20, \mathrm{~d}=8 \%$ ). The significant dual interaction between Sport Expertise and Exerted Effort indicates that the evolution of accurate response rate as a function of Exerted Effort differs for the collective and the fighting expert groups. As can be seen in (Figure 2), the evolution of the accurate response rate of SOC as a function of the intensity of the exerted effort is relatively complex, and it has an almost inverted $U$ shape, with a lower recognition rate at the rest condition and at the $\mathrm{E} 120 \%$ or $\mathrm{E} 100 \%$, and higher recognition rates at $\mathrm{E} 60 \%$ and $\mathrm{E} 80 \%$. In contrast, for $\mathrm{TKD}$, the evolution of the accurate response rate is almost linear, with the lowest rates at the rest condition and the highest at the E120\%, while the other exerted effort conditions (E60\%, E80\%, E100\%) induce progressive intermediary values. Further, the lack of significant triple interaction indicates that differences between the two groups of sport experts is observable in regard to their ability to differentiate between moderate- and highintensity facial-expression stimuli. 


\section{Reaction times}

Mean RT as a function of the manipulated factors is presented in Figures 2. The analysis revealed that experts in taekwondo $(\mathrm{F}(1,36)=5.9, \quad \mathrm{p}<0.02, \quad \hat{\omega}=0.66)$ required significantly less time $(\mathrm{M}=521 \mathrm{~ms} ; \mathrm{SD}=85 \mathrm{~ms})$ to recognize the different facial expressions than the experts in SOC $(\mathrm{M}=561 \mathrm{~ms} ; \mathrm{SD}=91 \mathrm{~ms})$. Overall, the participants recognized significantly more $(\mathrm{F}(1,36)=4.4, \mathrm{p}<0.05$, $\omega=0.52)$ moderate-effort facial expressions ( $\mathrm{M}=544 \mathrm{~ms} ; \mathrm{SD}=88 \mathrm{~ms}$ ) than intensive-effort facial expression $(\mathrm{M}=534 \mathrm{~ms} ; \mathrm{SD}=9 \mathrm{~ms})$. Choice reaction times also varied significantly as a function of $\mathrm{OEE}(\mathrm{F}(4$, $144)=15.5, p<0.001, \dot{\omega}=0.99)$. Overall, increased in the intensity of physical exercise exerted by participant was associated with a reduction in the time needed to recognize facial expressions.

The analysis did reveal a significant interaction between the factor Effort-related Facial Expression and $\operatorname{OEE}(F(4,144)<1)$; however, the dual interaction between the factors of Sport Expertise and Effort-related Facial Expression was significant $(F(1,36)=9.5$, $p<0.01, \omega=0.86$ ). The experts in taekwondo reacted faster to all facial expressions than SOC experts; however, it should be noted that this significant interaction suggests that the magnitude of the difference between the two groups was larger in terms of recognizing intensive facial expression pictures $\left(\mathrm{d}=26, \mathrm{ddl}=36, \mathrm{t}=52.45, p<0.001 ; \eta^{2}\right.$ $=0.144)$ than for recognizing low-intensity pictures $(\mathrm{d}=5, \mathrm{ddl}=36$, $\left.\mathrm{t}=54.9, p<0.001, \eta^{2}=0.298\right)$. The dual interaction between Sport Expertise and OEE was also significant $(F(4,144)=2.6, p<0.05$, $\omega=0.71$ ). Overall, the difference between the two groups in terms of the rapidity with which they recognized facial expression increased with increases in the intensity of the physical exercise.

Finally, this analysis revealed a triple significant interaction: Sport Expertise $\times$ Observer Exerted Effort $\times$ Effort Expression $(F(4$, $144)=3.6, p<0.01, \omega=0.87)$. To understand this interaction, we performed separate ANOVAs for moderate- and high-intensity facial expressions stimuli conditions; in the two ANOVAs, Sport Expertise (TKD vs. SOC) was used as a grouping factor and OEE (Rest, E60\%, $\mathrm{E} 80 \%, \mathrm{E} 100 \%$ and $120 \%$ ) as a repeated measure factor.

The analysis of the RT of the two groups in regard to facialexpression stimuli showing moderate-intensity effort revealed that a reliable effect was only found for factor $\operatorname{OEE}(F(4,144)=10.3, p<$ $0.001 ; \omega=0.99)$. For the two groups, increasing the intensity of the physical effort exerted was associated with a progressive reduction of CRT, with the longest CRT being exhibited during the rest period $(\mathrm{M}=565 \mathrm{~ms} ; \mathrm{SD}=87 \mathrm{~ms})$ and $\mathrm{E} 60 \% \quad(\mathrm{M}=594 \mathrm{~ms}, \mathrm{SD}=81 \mathrm{~ms})$, and the shortest during the E120\% condition $(\mathrm{M}=501 \mathrm{~ms} ; \mathrm{SD}=80 \mathrm{~ms})$. Nevertheless, the analysis did not reveal a significant effect for the factor of sport expertise $F(1,36)=2.2$, NS, nor a significant interaction between sport expertise and $\operatorname{OEE}(F(4,144)>1$, NS).

The result obtained from the analysis of the RT of the two groups in regard to intensive-effort facial expressions was completely different. The analysis revealed that both sport expertise $(F(1,36)=$ $10.5, p<0.01, \dot{\omega}=0.9)$ and $\operatorname{OEE}(F(4,144)=14.6, p<0.001 ; \dot{\omega}=0.99)$ had significant effects. Finally, the analysis also showed a significant dual interaction: Sport Expertise $\times \operatorname{OEE}(F(4,144)=4.9, p<0.001$; $\omega=0.96)$. As can be seen in Figures 2, this interaction indicates that the advantage of fighting experts over collective sports experts in terms of the recognition of facial expressions increases with increases in the intensity of physical effort produced.

\section{Discussion}

The aim of the present study was to evaluate the effect the short and long-term impacts of physical exercise have on the ability to recognize through facial expressions the degree of effort an actor is producing. In the study, short-term influences related to the effect created by the intensity of the physical effort participants exerted, whereas long-term influences were inherent in their expertise in their sport (fighting vs. collective sport). We also attempted to verify if practicing a combat sport improves the mechanisms of motor resonance and, consequently, facial recognition of the fatigue exposed on the face of an opponent. As we did not find any significant differences between the two groups in terms of their physiological measures (Table 1), we will focus our discussion on the impact of the long-term and short-term effects of sport practice on facial effort recognition.

\section{Effect of acute intensification of physical effort (short- term effect)}

The obtained results clearly reveal that acute, short-term physical exercise has a large effect on facial recognition performance. Increases in the effort exerted by the participants were almost systematically associated with regular increases in correct response rate and with regular reductions in correct response latencies. Shorter RT and higher correct response rates were obtained when higher-intensity efforts were produced, and vice versa for lower-intensity efforts. This pattern of results corroborates the well-documented enhancement of cognitive functions with increases in exercise intensity. ${ }^{24}$ Several studies have shown that, compared to low-intensity activity or a resting state, a high level of aerobic exercise induces an increase in BNDF concentration, which positively affects cognitive function. ${ }^{25,26}$ It is also consistent with Davey's exercise-arousal cognition-interaction theory, ${ }^{12}$ which predicts a linear relation between effort intensity, arousal level, and cognitive performance.

In addition, our study revealed some subtle effects that may contribute to the modification of the motor resonance condition. Undoubtedly, the highest levels of physical exercise (E120\%) induced an enhancement in the participants' ability to recognize all types of facial expressions. It must be noted, however, that the greatest enhancement was detected when the participants observed images of athletes' faces expressing higher levels of physical effort.

Motor resonance pattern seems to increase during episodes of high-intensity effort, but not when participants exert lower levels of effort. It is also interesting to note that this pattern concerning the effect of the intensity of physical exercise was more pronounced in the TKD than in the SOC group. As will be discussed in next section, the fact that fighting-sport experts are required to be more sensitive to facial expression than collective sports experts may support the finding that facial recognition performance is enhanced during intensive physical conditions. A similar pattern concerning the enhancing of motor resonance during higher efforts than lower efforts was also obtained in a relatively recent study, ${ }^{27}$ the authors used functional magnetic resonance imaging (fMRI) to examine brain responses to counterfactual statements concerning actions requiring high or low physical effort and compared them to equivalent factual statements describing the same actions. The results of their study revealed that the inferior parietal lobule, known to be the substrate of goal-directed action and an element of the motor resonance system, was more strongly stimulated during the presentation of statements 
related to high-effort than for low-effort statements.

The obtained results revealed that a specific effect relating to high-intensity physical exercise, but not lower level, enhanced the participants' recognition of effort-related facial expressions. Thus, the contribution of motor resonance to facial-recognition performance seems to be restricted to higher-intensity physical effort conditions.

\section{Effect of sport expertise (long-term effect)}

The aim of this study was also to evaluate the long-term effect of exercise on the ability to recognize facial expressions. We expected that expertise in sports that require the strategic, intensive processing of facial expressions would induce better recognition performance than sports that do not require such ability, and the obtained results were clearly in accordance with this expectation. Overall, the fightingexperts group exhibited higher correct response rates and shorter latencies in the facial recognition task than those in the collectivesport-experts group.

The lower performance of SOC in the facial recognition task confirms the findings of preceding works showing that higher performance in this sport does not rely on intensive decoding of facial expressions but on intense attention focus on lower parts of the opponent's body (hip, knee, ankle) as well as on the ball position. ${ }^{28,29}$ Further, the better performance of the TKD experts group in the facial-recognition task is also in accordance with other recent works. The recent study of Ruiz-Perez et al. ${ }^{30}$ indicates that taekwondo practitioners observe the trunk and head of their opponent in an attempt to predict their future actions. Moreover, Milazzo et al. ${ }^{31}$ also concluded that decoding body postures represents a crucial ability in taekwondo. The present study confirms and extends these results by showing that expertise in a fighting sport such as taekwondo clearly enhances athletes' ability to decode facial expressions linked to effort.

Our results also revealed that expertise in fighting sports also produces subtle effects. The superiority of TKD over SOC in terms of facial recognition was maximal under the condition during which the participants were exerting the highest intensity of physical effort $(120 \%)$ and were concurrently required to rapidly and accurately identify the facial expressions of athletes performing similar higherintensity efforts. Presumably, the reason TKD experts performed so favorably under these two combined conditions may be related to their experience of higher-intensity fighting situations, ${ }^{32}$ in which fast recognition of opponents' physical states is a crucial element. In a related study, Santos et al. ${ }^{21}$ examined 23 TKD games during the Beijing 2008 Olympic Games. They showed that the average ratio of attack time to assault preparation time was $\sim 1: 7$, but that the intensity of combat increased during round 3 , with the ratio being significantly reduced to $\sim 1: 5$. These results proved that the later stages of taekwondo fights require much greater effort than the first and second rounds; this is because during the third round TKD fighters experience accumulated fatigue from the previous two rounds and, concurrently, they are obliged to maintain a more intense rhythm than that applied at the beginning of the match. These findings suggest that the physiological demands of high-level taekwondo practice are based on the aerobic and the anaerobic galactic processes. ${ }^{23}$ Our results are consistent with those obtained by Santos et al. $;^{19}$ at the maximum intensity, the TKD fighters were able to respond quickly and with great precision. This demonstrates that the TKD practitioners had adapted to producing very intense efforts and responding rather quickly. Our results also accord with those of other studies; for example, Mang et al. ${ }^{33}$ found that high-intensity aerobic exercise induces increased plasticity in the M1 area.

It is also important to highlight the effect of the space of the game arena on facial-recognition capacity. According to the rules of the World Taekwondo Federation (WTF), the surface of the game mat is $64 \mathrm{~m}^{2}(8 \mathrm{~m} \times 8 \mathrm{~m})$, and each time a fighter leaves this combat area they are given a warning (the deduction of a point). Therefore, TKD fighters are face-to-face with their opponents throughout the duration of the competition, which lasts eight minutes (two minutes $\mathrm{x}$ three rounds, with a one-minute rest period between each round). This faceto-face confrontation throughout the competition allows the TKD fighter to adapt to the facial expressions exhibited by their opponent during the match, which gives TKD fighters an advantage in terms of learning, reading, and interpreting such facial expressions. On the other hand, a soccer field has a minimum area of $4050 \mathrm{~m}^{2}$, in which SOC players are mainly required to follow the movement of the ball and the placement of teammates and opponents in order to be able to predict future actions; hence, they have few opportunities to directly read the facial expressions of their opponents. ${ }^{19,34}$ In their study, Meier et al. ${ }^{16}$ suggest that structural adaptations are sport specific and are manifested in cerebral areas associated with the neuronal treatment of sport-specific-skills, which means that long-term sport practice can modulate cerebral plasticity in order to fit the specificities of the practiced sport.

The concept of motor resonance is a complex one, and it has been proposed to occur without conscious effort nevertheless this does not mean that everyone resonates with just anyone in any situation. ${ }^{35,36}$ Hence, the findings of the current study may be subject to other moderating effects such as individual characteristics. Indeed, research in social neuroscience and social cognition revealed numerous group biases in the processing of others' internal states, participants show less neural activity in areas for social perception in response to outgroup members and they hardly interpret their facial expressions than when observe in group members. ${ }^{37,38}$ These details must be considered when using the results of this study.

\section{Conclusion}

Taken together, the obtained results suggest plastic modification of the motor resonance phenomenon is caused through long-term practice in fighting sports. Further, the long-term effect of expertise seems also to mediate the short-term effects of the intensity of the physical exercise. It is likely that experts in TKD have learned to benefit from the increased motor resonance induced when they exert a high-intensity effort, allowing them to observe and recognize the facial movements of the opponent throughout the duration of a match.

Our results may have two main implications. The first implication is theoretical: the plastic modification caused as a result of developing the ability to decode facial expressions through long-term practice in TKD is probably sustained through cerebral plasticity; future neuropsychological studies involving functional imagery techniques may be able to precisely identify the cerebral structures involved in such modification. The second implication is practical: training methods in TKD sports rarely focus on developing athletes' abilities to decode facial expressions. The present study suggests that this ability is a critical property of expertise in such sports. Inviting athletes to pay more attention to decoding facial expressions may positively contribute to the enhancement of their performance. It should be mentioned that this study has some limitations such as the small size 
of groups and the limited number of repetitions. However, it must be mentioned that no study has made the link between physical effort and motor resonance has been published.

\section{Acknowledgements}

We would like to thank all those who participated in study, as well as the college and the ISSEP teachers for their valuable help in performing this study.

\section{Conflict of interest}

The authors have no conflicts of interest to declare.

\section{Ethical approval}

All procedures were approved by the University Research Ethics Committee and were conducted in accordance with the Declaration of Helsinki.

\section{Informed consent}

Informed consent was obtained from all individual participants included in the study.

\section{References}

1. Ploughman M. Exercise is brain food: the effects of physical activity on cognitive function. Dev Neurorehabil. 2008;11(3):236-240.

2. Ng QX, Ho CY, Chan HW, et al. Managing childhood and adolescent attention-deficit/hyperactivity disorder (ADHD) with exercise: A systematic review. Complement Ther Med. 2017;34:123-128.

3. Gallese V, Goldman A. Mirror neurons and the simulation theory of mindreading. Trends Cogn Sci. 1998;2(12):493-501.

4. Gallese V. Before and below 'theory of mind': embodied simulation and the neural correlates of social cognition. Philos Trans $R$ Soc Lond B Biol Sci. 2007;362(1480):659-669.

5. Agnew ZK, Bhakoo KK, Puri BK. The human mirror system: a motor resonance theory of mind-reading. Brain Res Rev. 2007;54(2):286-293.

6. Wicker B, Keysers C, Plailly J, et al. Both of us disgusted in My insula: the common neural basis of seeing and feeling disgust. Neuron. 2003;40(3):655-664.

7. Budde $\mathrm{H}$, Wegner $\mathrm{M}$, Soya $\mathrm{H}$, et al. Neuroscience of Exercise: Neuroplasticity and its Behavioral Consequences. Neural Plast. 2016;3.

8. Guizani MS, Tenenbaum G, Bouzaouach I, et al. Information-processing under incremental levels of physical loads: comparing racquet to combat sports. J Sports Med Phys fitness. 2006;46(2):335-343.

9. Rendi M, Szabo A, Szabo T. Relationship between physical exercise workload, information processing speed and affect. Int J Applied Sport Sci. 2007;19(1):86-95.

10. Davey CP. Physical exertion and mental performance. Ergonomics. 1973;16(5):595-599.

11. Niedenthal PM, Mermillod M, Maringer M, et al. The Simulation of Smiles (SIMS) model: Embodied simulation and the meaning of facial expression. Behav Brain Sci. 2010;33(6):417-433.

12. Kim YT, Seo JH, Song HJ, et al. Neural correlates related to action observation in expert archers. Behav Brain Res. 2011;223(2):342-347.

13. Proverbio AM, Crotti N, Manfredi M, et al. Who needs a referee? How incorrect basketball actions are automatically detected by basketball players' brain. Sci Rep. 2012;2:883.
14. Merino BC, Ehrenberg S, Leung D, et al. Experts see it all: configural effects in action observation. Psychol Res. 2010;74(4):400-406.

15. Aglioti SM, Cesari P, Romani $M$, et al. Action anticipation and motor resonance in elite basketball players. Nat Neurosci. 2008;11(9):1109-1116.

16. Meier J, Topka MS, Hänggi J. Differences in cortical representation and structural connectivity of hands and feet between professional handball players and ballet dancers. Neural plast. 2016.

17. Zoudji B, Thon B, Debû B. Efficiency of the mnemonic system of expert soccer players under overload of the working memory in a simulated decision-making task. Psychol Sport Exerc. 2010;11(1):18-26.

18. Harling SA, Tong RJ, Mickleborough TD. The oxygen uptake response running to exhaustion at peak treadmill speed. Med Sci Sports Exerc. 2003;35(4):663-668.

19. Santos VG, Franchini E, Silva LAE. Relationship between attack and skipping in taekwondo contests. J Strength Cond Res. 2011;25(6):1743-1751.

20. Campos FA, Bertuzzi R, Dourado AC, et al. Energy demands in taekwondo athletes during combat simulation. Eur J Appl Physiol. 2012;112(4):1221-1228.

21. Pomportes L, Davranche K, Brisswalter I, et al. Heart rate variability and cognitive function following a multi-vitamin and mineral supplementation with added guarana (Paullinia cupana). Nutrients. 2014;7(1):196-208.

22. Chamari K, Hachana Y, Ahmed YB, et al. Field and laboratory testing in young elite soccer players. Brit J of Sports Med. 2004;38(2):191-196.

23. Lautenbach F, Laborde SJP, Putman P, et al. Attentional distraction by negative sports words in athletes under low-and high-pressure conditions: Evidence from the sport emotional Stroop task. Sport Exerc Perform Psychol. 2016;5(4):296-307.

24. Davranche K, Brisswalter J, Radel R. Where are the limits of the effects of exercise intensity on cognitive control? J Sport Health Sci. 2015;4(1):56-63.

25. Hötting K, Schickert N, Kaiser J, et al. The effects of acute physical exercise on memory, peripheral BDNF and cortisol in young adults. Neural Plast. 2016.

26. Thomas R, Beck MM, Lind RR, et al. Acute exercise and motor memory consolidation: the role of exercise timing. Neural Plast. 2016.

27. Urrutia M, Gennari SP, Vega DM. Counterfactuals in action: an fMRI study of counterfactual sentences describing physical effort. Neuropsychologia. 2012;50(14):3663-3672.

28. Abreu A. Action anticipation in sports: A particular case of expert decision-making. Trends Sport Sci. 2014;21(1):5-11.

29. Savelsbergh GJ, Williams AM, Kamp JVD, et al. Visual search, anticipation and expertise in soccer goalkeepers. $J$ Sports Sci. 2002;20(3):279-287.

30. Perez LMR, Mendez RP, Manzano JAN, et al. Analysis of the visual behavior of taekwondists of different skills level. Rev Mex Psicol. 2013;30(1):32-40.

31. Milazzo N, Rosnet É, Fournier J. Stratégies de recherche visuelle et expertise décisionnelle en taekwondo. Mov Sport Sci. 2016;1(91):65-75.

32. Bouhlel E, Jouini A, Gmada N, et al. Heart rate and blood lactate responses during Taekwondo training and competition. Sci Sports. 2006;21(5):285-290.

33. Mang CS, Brown KE, Neva JL, et al. Promoting motor cortical plasticity with acute aerobic exercise: a role for cerebellar circuits. Neural Plast. 2016. 
34. Hughes M, Franksm I. Analysis of passing sequences, shots and goals in soccer. J Sports Sci. 2005;23(5):509-514.

35. Rizzolatti G, Fadiga L, Gallese V, et al. Premotor cortex and the recognition of motor actions. Brain Res Cogn Brain Res. 1996;3(2):131-141.

36. Rizzolatti G, Craighero L. The mirror-neuron system. Annu Rev Neurosci. 2004;27:169-192.
37. Gutsell JN, Inzlicht M. Using EEG mu-suppression to explore group biases in motor resonance. In: Derks B, Dan S, Ellemers. editors. Neuroscience of Prejudice and Intergroup Relations. Psychology Press, USA; 2013. p. 279-297.

38. Mathur VA, Harada T, Lipke T, et al. Neural basis of extraordinary empathy and altruistic motivation. Neuroimage. 2010;51(4):1468-1475. 